\title{
ON THE PRODUCT OF CONTINUOUS PRIME NUMBERS
}

\section{SHICHUN YANG AND QUNYING LIAO}

Abstract. Basing on some deep conclusions of the prime number estimation, we give a fine from of the product of continuous prime numbers. These conclusions further improve the corresponding inequality of Panaitopol and more accurate conclusion is obtained.

Mathematics subject classification (2010): Primary 11A41; Secondary 11N05.

Keywords and phrases: Continuous prime numbers, inequalities, the estimate of prime number.

\section{REFERENCES}

[1] R. J. BetTs, Using Bonse's inequality to find upper bounds on prime geps, J Integer Seq., 2007, 10 (3): 1-7, Article 07.3.8.

[2] D. Berkane And P. Dusart, On a constant related to the prime counting function, Mediterr J Math., 2016, 13: 929-938.

[3] H. Bonse, Üer eine bekannte eigenschaft der zahl 30 und ihre verallgemeinerung, Arch Math Phys, 1907, 12: 292-295.

[4] P. DUSART, Autour de la fonction qui compte le nombre de nombres premiers, Thèese, Université de Limoges, 1998, 172 pp.

[5] P. DUSART, Explicit estimates of some functions over primes, Ramanujan J., 2018, 45 (1): 227-251.

[6] H. Iwata, On Bonse's theorem, Math Rep Toyama Univ., 1984, 7: 115-117.

[7] C. LÁszLó, Generalized integers and Bonse's theorem, Studia Univ Babeş-Bolyai Math., 1989, 34 (1): 3-6.

[8] S. E. Mamangakis, Synthetic proof of some prime number inequalities, Duke Math J, 1962, 29: $471-473$.

[9] J. P. Massias AND G. RoBin, Bornes effectives pour certaines fonctions concernant les nombres premiers, J. Théor. Nombres Bordeaux, 1996, 8 (1): 215-242.

[10] L. PAnaitopol, An inequality involving prime numbers, Univ. Beograd Publ. Elektrotehn. Fak. Ser. Mat., 2000, 11: 33-35.

[11] L. PósA, Über eine Eigenschaft der Primzahlen (Hungarian), Mat. Lapok 11 (1960), 124-129.

[12] H. Rademacher And O. Toeplitz, The enjoyment of mathematics, Princeton Univ. Press, 1957.

[13] S. REICH, On a problem in number theory, Math Mag., 1971, 44: 277-278.

[14] G. RoBin, Estimation de la fonction de Tschebyshev $\theta$ sur le k-ieme nombre premier et grandes valeurs de la fonction $\omega(n)$ nombre des diviseurs premier de n, Acta. Arith., 1983, 42 (4): 367-389.

[15] J. B. Rosser AND L. SChOENFELD, Approximate formulas for some functions of prime numbers, Illinois J. Math. 1962, 6: 64-94.

[16] J. SANDóR, Über die Folge der Primzahlen, Mathematica (Cluj) 30, 1988, (53): 67-74. 